\title{
ENGINEERING INSPECTION ASSOCIATED ARTIFICIAL INTELLIGENCE FOR APPRAISAL OF THE PROPERTY IN NITEROI, RIO DE JANEIRO, BRAZIL
}

\author{
Surgelas Vladimir, Arhipova Irina, Pukite Vivita \\ Latvia University of Life Sciences and Technologies
}

\begin{abstract}
The construction sector is linked to the general development of a country. There is a lot of data scattered and not properly explored in relation to the buildings constructed. However, if these scattered data on the behavior of the real estate market are organized, combined with knowledge of civil engineering, this merger of information can mitigate some evaluation problems, especially those that are overvalued for unknown or dubious reasons. Thus, there is a need for models capable of working with limited data to analyze the causal relationships between explanatory variables and sales prices and, from there, predict property values. The purpose of this article is the innovative use of simple building inspection strategies to predict the market price for residential apartments. For this, 19 samples of residential apartments are used in the city of Niterói, Rio de Janeiro, Brazil, in February 2021. The methodology uses the results of the survey of civil engineering and converts them into heuristic terms predicting the price of the property. With this, the imprecision, uncertainty, and subjectivity of human expression combined with the knowledge of civil engineering result in a plausible solution and easy application in the market. Finally, the use of fuzzy logic in the evaluation of properties is an adequate unconventional method, in addition to avoiding repetition in regression coefficients in binary logic. To check the reliability of the method, the comparison between the market values of the samples and the values predicted by the fuzzy logic is used. The result according to the mean absolute percentage error (MAPE) can be interpreted as a good result (7\%).
\end{abstract}

Keywords: appraisal, artificial intelligence, civil engineering, ambiguous logic, inspection

\section{Introduction}

When examine the heuristic approach applied to the associated real estate market, a range of dispersed data not properly treated is found, which could somehow generate knowledge. Because, dispersed data is useless, but aggregated data can generate rules, rules can generate forecasts, forecasts generate knowledge, and knowledge generates income and increases the added value for the civil engineering sector and consequently leverages the economy in general. Therefore, the integration of different types of information and the recognition that the environment is constantly evolving creates the need to know how information is created, handled, and transmitted (Delgado et al., 2020). Based on that, the use of technology must be seen as a fundamental tool, meeting this constantly evolving scenario. In this sense, machine learning can be used in Civil Engineering (Manu, 2019). However, some precautions must be taken to consider a successful task. Thus, when the dispersed data becomes knowledge, there will be positive aspects for science, education, economics, job and income generation, social (ILO, 2018). Moreover, the methodology with Fuzzy Logic techniques is a weighted inference method that allows users to insert inaccurate data and heuristic observations and draw correct conclusions (Jochheim-Wirtz, 2013). In addition, the real estate market involves human expression heuristics affecting the property valuation by these heuristic variables (Renigier-Biłozor et al., 2021). However, if added to inspection criteria based on the technical knowledge of civil engineering, combined with rules of association of artificial intelligence, fuzzy logic, and heuristic perception, it results in the correct prediction of apartment prices, without the occurrence of regression instability. Moreover, the mining tools and applications make the scientific community pay attention to engineering, this can bring fascinating results (Arvindhan, Prasanna, 2016). From this, this fuzzy methodology becomes ideal for situations in which it is necessary to consider subjective aspects that are difficult to measure. As well as those aspects inserted as the subjective value of a property. Another aggravating factor is related to the existing abnormality in the building, useful life, and engineering systems involved (Abbott et al., 2007). In this context, the physical state of the building reflects its functional performance (NEN 2, 2006). Thus, that progressive deterioration of buildings has damaged the urban image of Portuguese cities and afflicted the habitability of many rented units (Pedro et al., 2008). Consequently, this status of conservation is reflected in the market price. Furthermore, the lack of inspection of buildings in buildings can result in an early loss of engineering systems (Syamilah et al., 2016). So, the earlier an anomaly is detected in a building, the more efficient and less costly the intervention in the future will be (Che-Ani et al., 2015). In this way, in order to make a diagnosis of a pathological manifestation in the building, it is necessary 
to carry out, initially, a visual inspection to make a collection of data, apparently dispersed (Garrido Vasquez et al., 2016).On the other hand (Mansuri, Patel, 2021) demand the automated use of building inspections. Alleging a decrease in human errors at the time of inspection. Anyway, some precautions must be followed in order to avoid errors in the inspection. Thus, it is necessary to follow some basic procedures, such as:

a) Inspection for mapping symptoms. This procedure begins with a visual inspection, where the aim is to identify the symptoms of existing anomalous in the structure through a mapping of the evidence;

b) Collection of data and scattered information. Furthermore, in several countries, the physical condition of a building is assessed and inspected based on the diagnosis of the degree of deterioration of the building elements (Costa Branco De Oliveira Pedro, et al., 2008). The Portuguese method of building inspection allowed for an extraordinary update of the lease agreements signed in that country. To that proposal, the National Civil Engineering Laboratory (LNEC) developed the "method to assess the state of conservation of buildings" within the legal framework of Portugal (Pedro et al., 2009). The same authors saws that the resource with only visual inspection was considered adequate because it allows the detection of the most notorious anomalies and at the same time contributes to the balance of time and resources with the low cost of the process. The method allows converting the anomaly index (expressed on a continuous scale from 1.00 to 5.00). That scale reflects the status of conservation of the under appraisal (expressed in a nominal scale from poor to excellent). For practical purposes, anomalies in buildings are classified into 5 basic categories. 1) Very light (excellent), 2) Light (good), 3) Medium (average), 4) Serious (bad), 5) Very serious (terrible). The anomaly index (AI) building's conservation status are: very light 5.00 $\geq \mathrm{AI} \geq 4.50$; Light $4.50>\mathrm{AI} \geq 3.50$; Medium $3.50>\mathrm{AI} \geq 2.50$; Serious $2.50>$ $\mathrm{AI} \geq 1.50$; very serious $1.50>\mathrm{AI} \geq 1.00$ (Pedro et al., 2008). So, heuristics are ideal for solving poorly structured problems (Abel, 2003). In this context, they also provide acceptable solutions to complex problems, if supported by artificial learning algorithms. This cognitive heuristic form influences the decision, which starts to be made based on a balance between reasons for and against the various alternatives. This source of discovery of motives occurs in situations of uncertainty, conflict (Tversky, Kahneman, 1974). Thus, among the most important characteristics of the property, in the particular case of the real estate market (Libby, Kyle, 2014) it has some differentiating characteristics, such as strong influences on the location and heterogeneity of assets, in addition to operating under imperfect competition. So, the purpose of this article is to use simple building inspection strategies to predict the market price for residential apartments. For this, only 19 samples of residential apartments are used in the city of Niterói, Rio de Janeiro, Brazil in February 2021. Thus, the process of appraisal follows by the Fuzzy method combined with the incorporation of the association rules originated from Weka "apriori" algorithm (Witten et al. 2011). In Mandami defuzzification process, the interpretation of the ambiguous set of output inferred carried out, to obtain a numerical value. So, the InFuzzy software was chosen to discover the prices of the residential flats. To verify the reliability of the method, a comparison between the market values of the samples and the values predicted by the fuzzy logic was used, by the mean absolute percentage error (MAPE).

\section{Materials and Methods}

This experiment uses the samples with only 19 residential apartments in Niterói, Rio de Janeiro in February 2021. So, for a better understanding of the methodology used it was divided into 5 phases described below. Phase 1 - selecting scattered data, Phase 2 - Preparing files to association rules, Phase 3 - Generating association rules, Phase 4 - Fuzzy Inference process, Phase 5 - Interpretation of the precision of the experimental model. So, detailing the phases.

Phase 1 - Search for the data source available on the World Wide Web. The variables evolved are the dependent variable $\mathrm{Y}$ is housing price (Euro per $\mathrm{m}^{2}$ ) and following factors or independent variables were chosen: AREA $\left(\mathrm{m}^{2}\right), \mathrm{CONSV}$ AP $=$ apartment conservation (index from inspection), CONSV_BUILD = building conservation (new (1), renewed (2), old (3)), ROOMS = number of rooms (1), (2), (3), (4); PARKING = car parking (inside build (1), outside build (2), no parking(3)), AIR CONDITIONING = comfortable (direct (1), uncomfortable (2)), VIEW = environmental / OCEAN VIEW (direct (1), no direct (2)). Performs a practical survey of the real estate market in the city of Niterói in Rio de Janeiro, Brazil and collects scattered data from this real estate market. This is related to offers for the sale of 1 , 2, 3 and 4 rooms residential apartments. Determines the heuristic variables to be used in the evaluation process.; Analyzes the characteristics of these apartments using civil engineering inspection techniques.; It describes the state of the apartment's degree of anomaly according to the score (Table 1) obtained on 
the field application form (Pedro et al., 2008).; compiles all the dispersed data obtained in the civil engineering inspection and selected heuristic variables.

Phase 2 - The data is organized by the author to aggregate the attributes of each apartment. The author created the ARFF file on the Notepad++ with the content of the price/square meter ratio. The application of the ARFF file is developed by the author is later launched in the Weka software (Witten et al., 2011). Phase 3 - From the data obtained in phase 2, the use of artificial intelligence to obtain association rules begins. For that, data mining is necessary. Weka Software will generate association rules that will present a pattern of behavior (Steven et al., 2019), which in this case are related to the data collected from the property samples. For this task, the "apriori" algorithm was chosen. Apriori performs the mining of association rules. This algorithm is suitable for a small amount of data, ideal for this experiment.

Phase 4 - In this part, the InFuzzy software is used (Posselt et al., 2015). It is a tool for the development of diffuse system applications. In this task, the author inserts the association rules of interest chosen for this experiment, as selected in Step 3 which will insert in the InFuzzy software to generate association rules used in Fuzzy Logic. In this important phase, the price of the property is forecast, using the InFuzzy Software for modeling the diffuse system. The modeling of a tree is done using linguistic terms to generate a function per variable. From that, the methodology is based on ambiguous logic and precedes the following basic steps (Figure 1) Moreover, the range is chosen based on the experience of this researcher. In this way, the variables, scope, classification, parameter, and association function are defined. To define the parameter item in the area variable, the author chooses a Gaussian function, defined below. For this, was checking the relevance of the samples by Chauvenet's exclusion criterion. By this criterion, the sample is relevant if the quotient between its deviation (which is the difference in absolute value between the sample value and the mean) and the standard deviation is lower than the tabulated Chauvenet critical number. Applied statistical theory: where $t_{c}=$ percentile values for the distribution " $t$ " Student to " $n$ " samples and " $n-1$ " degrees of freedom with the confidence of $80 \%$. The confidence limit is defined by the model

Phase 5 - The purpose of this step is to interpret the precision of the experimental model. It is about reporting the results of step 4 and checking for compatibility. This compatibility and precision of these results are accomplished by the following metrics, the mean percentage absolute error (MAPE).

\section{Results and Discussion}

Phase 1 - selecting scattered data. 19 samples of apartments advertised on the World Wide Web are used to compose the learning of the "apriori" algorithm, 37 construction items are analyzed visually through a form, involving various specialties within the field of engineering science. The linguistic terms are introduced after the comparison with the information in Table 2 and after transcribed in Table 1 and Table 2. The Icarai beach at Niterói City 53\% roughly are residential flats in good condition, these are among new and reformed residential apartments.

Table 1

Abnormalities took from inspection (inside) (Source: author)

\begin{tabular}{|c|c|c|c|c|c|}
\hline \multirow[b]{2}{*}{ Variable } & \multicolumn{5}{|c|}{$\begin{array}{c}\text { Number of anomalies found after civil engineering inspection } \\
\text { inside of the apartment building }\end{array}$} \\
\hline & $\begin{array}{c}\text { Very light } \\
\text { (excellent) } \\
5.00>\text { IA } \geq 4.50\end{array}$ & $\begin{array}{c}\begin{array}{c}\text { Light } \\
(\text { good })\end{array} \\
4.50>\text { IA } \geq \\
3.50\end{array}$ & $\begin{array}{c}\text { Medium } \\
(\text { medium }) \\
3.50>\text { IA } \geq \\
2.50\end{array}$ & $\begin{array}{c}\begin{array}{c}\text { Serious } \\
\text { (bad) }\end{array} \\
2.50>\mathrm{IA} \geq \\
1.50\end{array}$ & 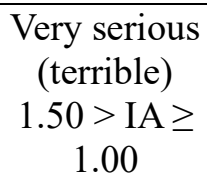 \\
\hline Consv ap & 9 & 10 & 0 & 0 & 0 \\
\hline
\end{tabular}


Table 2

Result of examination on residential flats in Niterói city (Source: author)

\begin{tabular}{|c|c|c|c|c|c|}
\hline & \multicolumn{3}{|c|}{ Variables } & \multirow[b]{2}{*}{$\begin{array}{l}\text { The linguistic term used } \\
\text { in "consv_ap" }\end{array}$} & \multirow[b]{2}{*}{$\begin{array}{l}\text { The linguistic term } \\
\text { used in "consv_build" }\end{array}$} \\
\hline & $\begin{array}{l}\text { Index: } \\
\text { consv_ap }\end{array}$ & Area $\left(\mathrm{m}^{2}\right)$ & $\begin{array}{c}\text { Index: } \\
\text { consv_build }\end{array}$ & & \\
\hline 1 & 4,79 & 70,00 & 1.00 & excellent & new \\
\hline 2 & 3,96 & 85,00 & 3,00 & good & old \\
\hline 3 & 3,95 & 100,00 & 3,00 & good & old \\
\hline 4 & 4,33 & 90,00 & 1,00 & good & new \\
\hline 5 & 4,34 & 88,00 & 3,00 & good & old \\
\hline 6 & 4,01 & 180,00 & 3,00 & good & old \\
\hline 7 & 4,63 & 110,00 & 1,00 & excellent & new \\
\hline 8 & 4,79 & 200,00 & 2,00 & excellent & renewed \\
\hline 9 & 4,03 & 240,00 & 1,00 & good & old \\
\hline 10 & 4,07 & 94,00 & 1,00 & good & old \\
\hline 11 & 4,08 & 95,00 & 1,00 & good & old \\
\hline 12 & 4,52 & 372,00 & 1,00 & excellent & new \\
\hline 13 & 4,57 & 180,00 & 1,00 & excellent & new \\
\hline 14 & 4,56 & 250,00 & 1,00 & excellent & new \\
\hline 15 & 4,60 & 91,00 & 1,00 & excellent & new \\
\hline 16 & 4,66 & 150,00 & 2,00 & excellent & renewed \\
\hline 17 & 4,19 & 90,00 & 1,00 & good & old \\
\hline 18 & 4,51 & 93,00 & 1,00 & excellent & new \\
\hline 19 & 4,63 & 94,00 & 1,00 & excellent & new \\
\hline
\end{tabular}

Phase 2 - Preparing files to association rules. The author created the ARFF file on Notepad++ with the content of the price /square meter ratio. It was possible to analyze the purpose of price prediction in this first result, 90 best rules following $70 \%$ (confidence) were chosen. Figure 1 shows a partial view construction of the ARFF file extension. Thus, phase 3 starts only from the creation of this file.

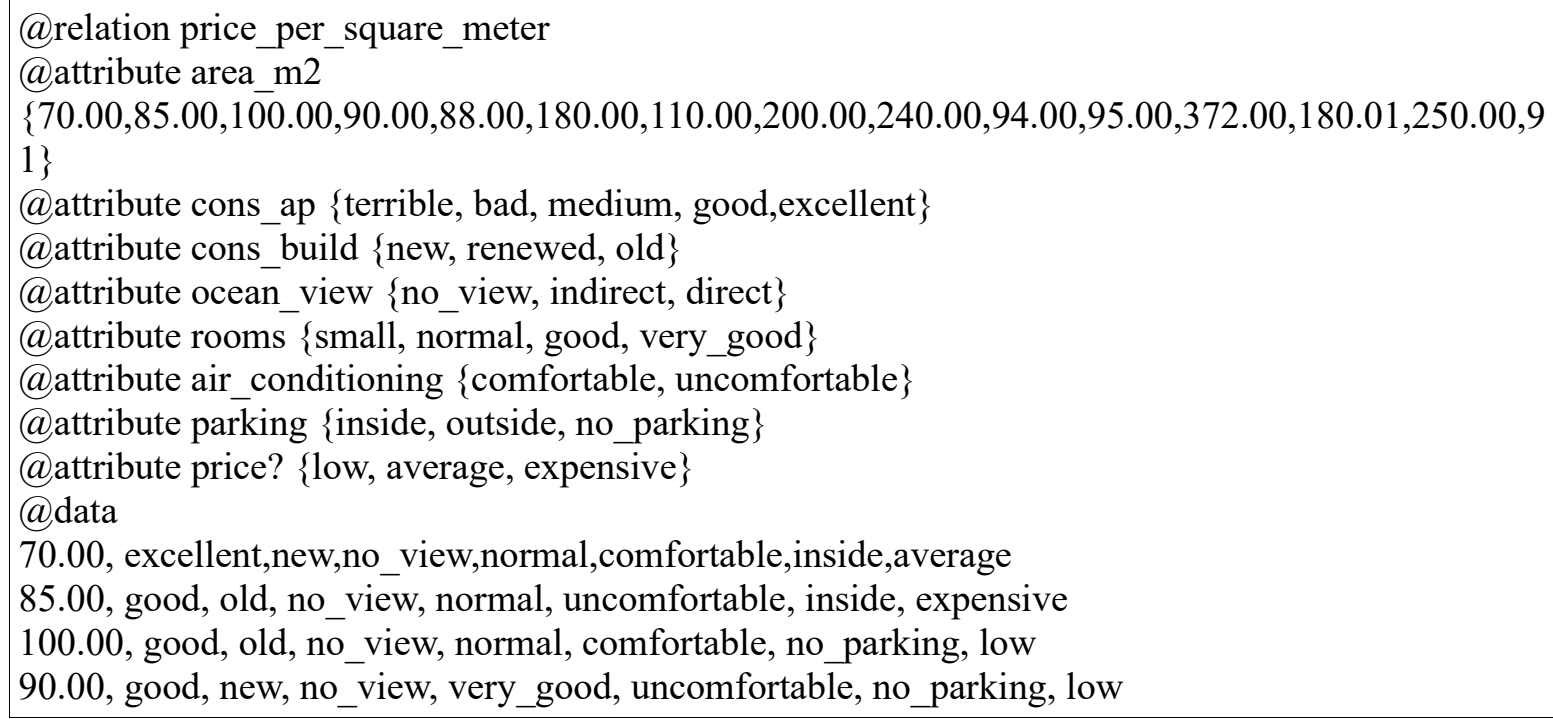

Figure 1. Partial view of ARFF file creates in Notepad++ (Source: author)

Phase 3 - Generating association rules. The file format required for the Weka software to understand is not obtained automatically. The domain of the attribute is described by the author. Thus, the Weka software generated 2,189 association rules. It occurs due to the learning/data mining algorithm called 
"apriori". The criterion established by the researcher was $70 \%$ confidence. After analyzing the purpose of the price forecast, 90 best rules were selected with the price as a consequence. In this context some of these rules are presented below.

IF (air_conditioning $=$ comfortable) AND $($ parking $=$ inside $) \quad$ THEN $\quad$ (Price $=$ average $)$

Phase 4 - Fuzzy Inference process. By the way, in this step, the price of the property is forecast, using the Infuzzy software for modeling the diffuse system. In the defuzzification activity. The rules's association from Weka were written in InFuzzy software. Fuzzy outputs are converted into discrete values needed to drive the control mechanism. The method of defuzzification used is the center of gravity. Sample number 12 was eliminated after verification by the exclusion criterion "Chauvenet". Chauvenet's criterion makes the assumption that the values in a dataset are normally distributed. Therefore, of the 20 samples were used. Nevertheless, only 19 remained. For this, the calculation below defines the maximum and minimum values, mean, the standard deviation of the variable "area". These values will be necessary to write the Gaussian function in the Infuzzy software. Where:
Calculation of sample average
$(\bar{x})=140.63 \mathrm{~m}^{2}$
The standard deviation of the sample.
$(S) \quad=78.81$
Sample - maximum area found
Max $\quad 372.00$
Sample - minimum area found
Min $\quad 70.00$
Sample numbers
$(n) \quad=19$
Degrees of freedom
$(n-1)=18($ with $80 \%$ confidence $)$
Percentile values for Student's t-distribution
$(t c)=1.33$ (table value)

Table 3

\begin{tabular}{|c|c|c|c|c|c|c|}
\hline \multicolumn{2}{|c|}{ Variables } & Range & Classification & \multicolumn{2}{|c|}{ Parameters } & Function \\
\hline \multirow{23}{*}{ In-put } & \multirow{3}{*}{ AREA } & \multirow{3}{*}[0,375]{} & low & 0.00 & 116.58 & Left ramp \\
\hline & & & normal & 140.63 & 78.81 & Gaussian \\
\hline & & & big & 164.68 & 375.00 & Right ramp \\
\hline & \multirow{5}{*}{ CONSV_AP } & \multirow{5}{*}[0,5]{} & terrible & 0.0 & 1.5 & Left ramp \\
\hline & & & bad & $1.5 / 2.0$ & 2.5 & Triangle \\
\hline & & & medium & $2.5 / 3.0$ & 3.5 & Triangle \\
\hline & & & good & $3.5 / 4.0$ & 4.5 & Trapezoidal \\
\hline & & & excellent & 4.5 & 5.0 & Right ramp \\
\hline & \multirow{3}{*}{ CONSV_BUILD } & \multirow{3}{*}[0,3]{} & new & & 1 & Discreet \\
\hline & & & renewed & & 2 & Discreet \\
\hline & & & old & & 3 & Discreet \\
\hline & \multirow{3}{*}{ OCEAN VIEW } & \multirow{3}{*}[0,3]{} & no view & 0.5 & 1.2 & Left ramp \\
\hline & & & indirect & $0.8 / 1.65$ & 2.5 & Triangle \\
\hline & & & direct & 2.1 & 3.0 & Right ramp \\
\hline & \multirow{4}{*}{ ROOMS } & \multirow{4}{*}[0,4]{} & small & & 1 & Discreet \\
\hline & & & normal & & 2 & Discreet \\
\hline & & & good & & 3 & Discreet \\
\hline & & & very good & & 4 & Discreet \\
\hline & \multirow{2}{*}{$\begin{array}{l}\text { AIR } \\
\text { CONDITIONING }\end{array}$} & \multirow[t]{2}{*}[0,2]{} & comfortable & & 1 & Discreet \\
\hline & & & uncomfortable & & 2 & Discreet \\
\hline & \multirow{3}{*}{ PARKING } & \multirow{3}{*}[0,3]{} & inside & & 1 & Discreet \\
\hline & & & outside & & 2 & Discreet \\
\hline & & & no parking & & 3 & Discreet \\
\hline \multirow{3}{*}{ Output } & \multirow{3}{*}{ PRICE } & \multirow{3}{*}[0,3200]{} & low & 150 & 1100 & Left ramp \\
\hline & & & average & $\begin{array}{l}700 / \\
1400\end{array}$ & 2200 & Triangle \\
\hline & & & expensive & 1900 & 3500 & Right ramp \\
\hline
\end{tabular}

Summary Fuzzy sets (Source: author)

Confidence limits were defined by the Chavenaut criterion, according to the following model. 


$$
X \operatorname{maxmin}=\bar{x} \pm t c \times\left(\frac{S}{\sqrt{n-1}}\right) \quad \begin{aligned}
& \mathrm{X}_{\max }=164.68 \mathrm{~m}^{2} \\
& \mathrm{X}_{\min }=116.58 \mathrm{~m}^{2}
\end{aligned}
$$

Table 3 shows a summary of Fuzzy sets. So, by the simple analysis of the conventional statistical operation, the parameters of the variable "area" found

Thus, filling the form with the rules is occupied with heuristic data brought by the investigator as reported at the time of the inspection of the building and the apartment. After that, the computer simulation begins. Figure 2 shows part of the output variable (price) and the result obtained by the reference point of the center of gravity.

\begin{tabular}{|cccccccc|}
\hline consv_ap & area & consv_build & ocean_view & room & air_conditioning & parking & "PRICE" \\
\hline 4.79 & 70.00 & 1.00 & 0.50 & 2.00 & 1.00 & 1.00 & $1,433.33$ \\
3.96 & 85.00 & 3.00 & 0.50 & 2.00 & 1.00 & 1.00 & $1,433.33$ \\
3.95 & 100.00 & 1.00 & 0.50 & 2.00 & 1.00 & 3.00 & 953.42 \\
4.33 & 90.00 & 1.00 & 0.50 & 4.00 & 2.00 & 3.00 & 372.64 \\
\hline
\end{tabular}

Figure 2. Partial view of the simulation on Infuzzy software (Source: author)

Next, figure 3 shows the results of the simulation in the Infuzzy software. The result using 19 samples to obtain a property price forecast was analyzed using the center of mass defuzification procedure. This shows the final variable of the process (Price at 1,433.33 Euro per $\mathrm{m}^{2}$ ).

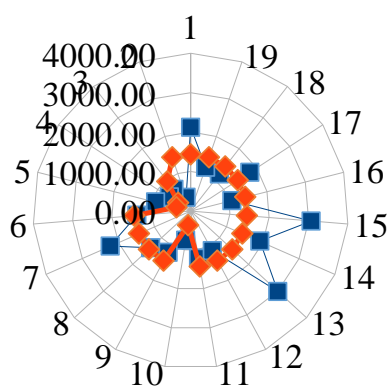

MAPE 58\%

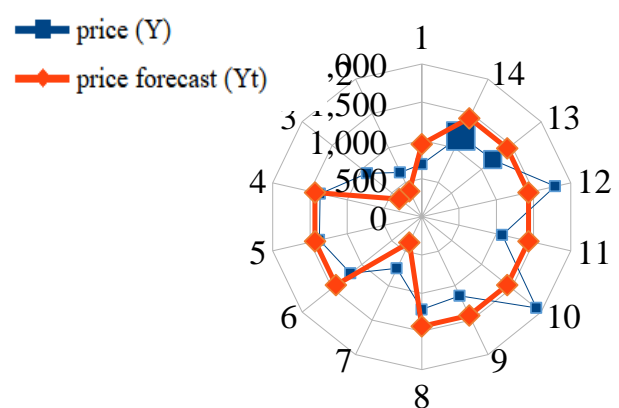

MAPE 7\%

Figure 3. The results get by the center of mass reference poin (Source: author)

However, when removing samples 1, 2, 7, 13, 15 for having the hotspot or heuristic effect of the influence of the ocean view (beach), there was a substantial improvement in the margin of error according to MAPE (7\%) (Table 4). This demonstrates that the method used meets the purpose for which it is intended. Thus, the price forecast adjusts better to the values offered in the market. The small variation is due to the effects of conditions and pathologies found at the time of building inspections. 
Summary MAPE (Source: author)

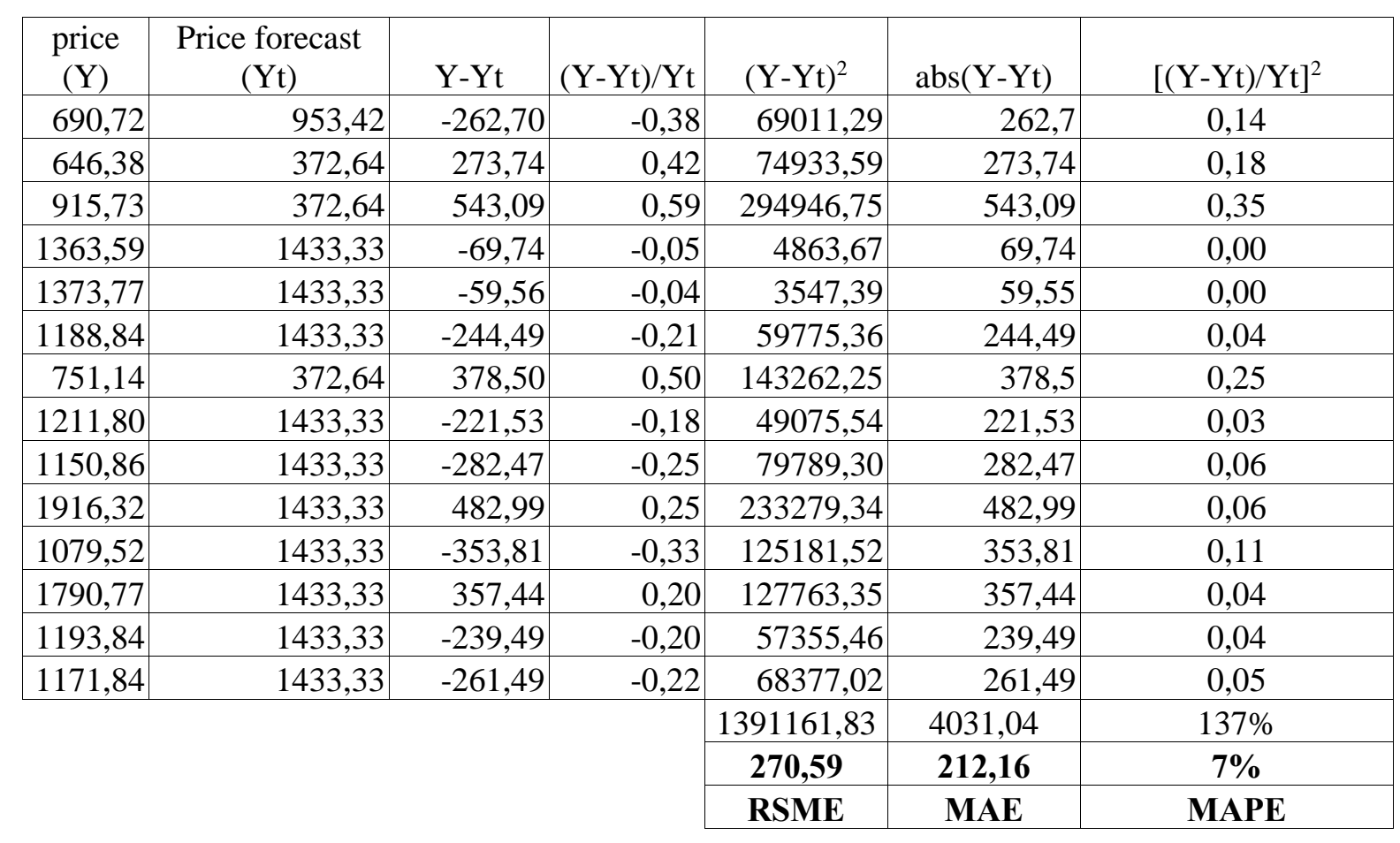

Phase 5 - Interpretation of the precision of the experimental model.

This research demonstrates that the heuristic knowledge of a civil engineering inspection process based on "in loco" experiments can be translated into a price estimation system with the aid of artificial intelligence tools. It should be added that the current proposal to improve the property evaluation process does not present the obstacles or the search for statistical strategies present in many cases in multiple regression. It should be added that the present evaluation method, as it has heuristic precepts, artificial intelligence and fuzzy logic associated with each other, does not present effects of multicollinearity or redundancy in the variables involved. Allied to the result as good or close to the conventional statistical process. In addition, this academic proposal has the advantage of establishing linguistic terms and parameters that are easy to handle and currently used by real estate appraisers, without the need to resort to complex statistical foundations such as those present in the multiple regression method. Table 3 provides the area parameters. The range of parameters for specific ratings overlaps a little, which can lead to errors if we try to predict the price using conventional statistics. However, in the proposed methodology, this possible error is absorbed by the elasticity promoted by the heuristic. In fact, after several tests eliminating extreme areas, the results of the apartment price forecast have remained unchanged. Because the focus here is correlated to the indexes derived from the standard form of construction inspection and to the precepts of civil engineering and to the reality of the value of the real estate market in the region under study. Thus, the study was not carried out on a larger data set, as it sought to feed the database of the artificial learning algorithm with few elements. The objective is to simulate the reality found by professional evaluators. In general, these professionals do not have, in practice, many similar samples to perform the evaluations. Therefore, it was thought to use about 20 samples in the same area as the study. From this, it was observed in this study the behavior of the methodology and the results obtained reflect a market equivalence in the forecast of the apartment price. The results of this stage 5 refer to the contrast of the market prices offered for the real estate samples with the values calculated and predicted by the ambiguous logic. The result achieved with the sending of error metrics to evaluate the forecasts with the absolute average percentage error (MAPE), was around $58 \%$. In some areas, for example, when working with human subjectivity, it is fully expected that forecast values will also have a high coefficient of variation in the data generation process. This does not mean that the forecast model is incorrect. For, several external factors may have interfered with the forecast. In other words, this apparently high value reveals, in fact, the existence of some samples with very high values in relation to the average of the same inquired offers. This is due to the location of 
these same samples. Some of them are located a few meters from the beach and have a direct view of the sea. This differential causes the value to rise a lot in relation to the average of the other samples of residential apartments. Thus, the average rate that the model is using in percentages for each forecast has an error rate of around 58\%. Thus, the Fuzzy model associated with the inspection techniques of civil engineering is better suited to the reality of prices in the city of Niterói. However, when the researcher removed samples 1, 2, 7, 13, 15 for having the hotspot or strong heuristic effect of the influence of the sea view (beach), there was a substantial improvement in the margin of error, and according to the MAPE it was only 7\% (seven). In addition to presenting a visual diagnosis on the state of conservation of the apartment and building. In this bias, if we compare the average value of the prices offered 1,433.91 euros per $\mathrm{m} 2$ with the average price of the fuzzy model 1,240.59 euros per $\mathrm{m} 2$, this represents a difference of only $15 \%$ between them. Thus, in the case of real estate offer values, this percentage of $15 \%$ can be understood as a discount to be applied to the offered value. This being a common form of negotiation, in practice exercised by the real estate market (Thomas et al., 2015).

\section{Conclusions}

The methodological analysis in Niterói concerns knowledge of civil engineering, artificial intelligence, and linguistic terms appropriate to the real estate market. Thus, the values obtained are very close to each other. The fuzzy process is better understood due to the ease with which linguistic terms are used in relation to the conventional statistical game. Furthermore, without presenting the drawback of multicollinearity. Therefore, with regard to the linguistic rules relevant to human behavior and the rules of association created by the "a priori" algorithm, this study concludes that the use of fuzzy logic in the evaluation of properties is an appropriate unconventional method, in addition to avoiding repetition in regression coefficients in the binary logic. The good consequences of the know-how were harmonious with the market value. Over time, the experiment acquires fabulous results in this first experiment carried out in the city of Niterói, Rio de Janeiro, Brazil.

\section{References}

1. Abel, C. F. (2003). Heuristics and Problem Solving. New Directions for Teaching and Learning. 53-58. DOI 10.1002/tl.113.

2. Abbott, G., McDuling, J., Parsons, S., Schoeman, J. (2007). Building condition assessment: a performance evaluation tool towards sustainable asset management. Viewed 29 April, 2021, (http://hdl.handle.net/10204/1233)

3. Arvindhan, M., Prasanna, S. (2016). Study on various techniques of text mining. International Journal On

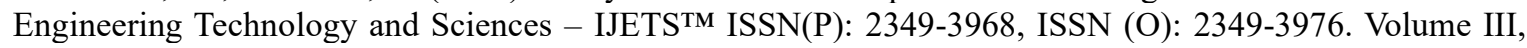
Issue $\mathrm{V}$, May

4. Che-Ani, A. I., Ismail, I., Johar, S., Abd-Razak, M. Z., Hamzah, N. (2015). Condition Survey Protocol: A system for building condition assessment. In Applied Mechanics and Materials (Vol. 747, pp. 347-350). Trans Tech Publications Ltd.

5. Costa Branco De Oliveira Pedro, J. A., Vasconcelos de Paiva, J., Vilhena, A. (2008). Portuguese method for building condition assessment. Structural survey 26 (4), 322-335.(2008). Viewed 30 April, 2021, (https://repository.tudelft.nl/islandora/object/uuid:8bb4f41f-1281-40c0-a881e3413330efba?collection=research )

6. Delgado J. M. D., Oyedele L., Demian P., Beach T. (2020). A research agenda for augmented and virtual reality in architecture, engineering and construction. Advanced Engineering Informatics, Volume 45, 2020, 101122, ISSN 1474-0346. Viewed 28 Arpril, 2021, (https://doi.org/10.1016/j.aei.2020.101122 ).

7. Faqih F., Zayed T. (2021). A comparative review of building component rating systems. Journal of Building Engineering, Volume 33, 2021, 101588, ISSN 2352-7102. Viewed 27 April, 2021, (https://doi.org/10.1016/j.jobe.2020.101588).

8. Garrido Vazquez E., Naked Haddad A., Linhares Qualharini E., Amaral Alves L., Amorim Féo I. (2016) Pathologies in Reinforced Concrete Structures. In: Delgado J. (eds) Sustainable Construction. Building Pathology and Rehabilitation, vol 8. Springer, Singapore. Viewed 26 April, 2021, (https://doi.org/10.1007/978-981-10-0651-7_10 ).

9. ILO, International Labour Office-Geneva. (2018). World Employment and Social Outlook 2018 - Greening with Jobs.

10. Jochheim-Wirtz, Christoph. (2013). The Official Real Estate Appraisal in Germany. Geonauka 01(03):1-5.

11. Libby W., Kyle M. (2014). Transaction Cost Economics and the Cognitive Perspective: Investigating the Sources and Governance of Interpretive Uncertainty. AMR, 39, 344-363, Viewed 29 April, 2021, (https://doi.org/10.5465/amr.2011.0463 ). 
12. Mansuri L. E., Patel D. A. (2021). "Artificial intelligence-based automatic visual inspection system for built heritage", Smart and Sustainable Built Environment, Vol. ahead-of-print No. ahead-of-print. Viewed 29 April, 2021, (https://doi.org/10.1108/SASBE-09-2020-0139).

13. Manu Mitra. (2019). Machine Learning in Civil Engineering. J Archit Sci Civ Eng. 2019; 1 (1): 1-2.

14. NEN, 2. (2006). Condition Assessment of Building and Installation Components. Dutch: NEN.

15. Pedro, J. B., Vilhena, A., Paiva, J. V., \& PINHO, A. (2009). Método de avaliação do estado de conservação de imóveis: Desenvolvimento e aplicação. (Method of Assessing the State of Conservation of Real Estate. Development and Application). Revista Engenharia Civil, (35), 57-73. Viewed 29 April, 2021, (http://www.civil.uminho.pt/revista/artigos/n35/Pag.57-74.pdf ). (in Portuguese)

16. Posselt, E., Frozza, R., Molz, R. (2015). INFUZZY: Ferramenta para desenvolvimento de aplicações de sistemas difusos (INFUZZY: Tool for developing diffuse system applications). Revista Brasileira de Computação Aplicada. 7, 1 (fev. 2015), 42-52. Viewed 29 April, 2021, (https://doi.org/10.5335/rbca.2015.3960 ). (in Portuguese).

17. Renigier-Biłozor, M., Janowski, A., Walacik, M. et al. Human emotion recognition in the significance assessment of property attributes. J Hous and the Built Environ (2021). Viewed 29 April, 2021, (https://doi.org/10.1007/s10901-021-09833-0 ).

18. Steven Lang, Felipe Bravo-Marquez, Christopher Beckham, Mark Hall, and Eibe Frank. (2019). WekaDeeplearning4j: a deep learning package for Weka based on DeepLearning4j. Knowledge-Based Systems, 178. Pages 48-50, ISSN 0950-7051. Viewed 28, April, 2021, (https://doi.org/10.1016/j.knosys.2019.04.013 ).

19. Syamilah Yacob, Azlan Shah Ali, and Au-Yong Cheong Peng. (2016). Building Condition Assessment: Lesson Learnt from Pilot Projects. The 4th International Building Control Conference 2016 (IBCC 2016). MATEC Web Conf. Volume 66. Viewed 30 April, 2021, (https://doi.org/10.1051/matecconf/20166600072 ).

20. Thomas, S. P., Manrodt, K. B., Eastman, J. K. (2015). The impact of relationship history on negotiation strategy expectations: A theoretical framework. International Journal of Physical Distribution \& Logistics Management, 45(8), 794-813.

21. Tversky, A., Kahneman, D. (1974). Judgment under uncertainty: Heuristics and biases. Science, 185, 11241131.

22. Witten, I. H., Eibe F., Hall. M. A. (2011). Data mining: practical machine learning tools and techniques. -3 rd ed. / Ian H. Witten,. - (The Morgan Kaufmann series in data management systems) ISBN 978-0-12-3748560 (pbk.), $\quad$ Viewed $30 \quad$ April, $\quad$ 2021, (https://www.wi.hswismar.de/ cleve/vorl/projects/dm/ss13/HierarClustern/Literatur/WittenFrank-DM-3rd.pdf ).

Information about authors:

Vladimir, Surgelas, Ph.D student at Latvia University of Life Sciences and Technologies. dr.engenho@gmail.com Irina, Arhipova, Vice-Rector of Sciences at Latvia University of Life Sciences and Technologies. zinpror@1lu.lv Vivita, Pukite, Dr.oec., Professor, Latvia University of Life Sciences and Technologies, Latvia. vivita.pukite@1lu.lv 